\section{AB0031 METABOLIC ADAPTATION OF HUMAN NEUTROPHILS TO GLUCOSE DEPRIVATION IS IMPAIRED WITH INCREASING AGE}

M. Pfeiffenberger ${ }^{1,2}$, P. L. Krauß ${ }^{1,2}$, T. Buttgereit ${ }^{3}$, Y. Chen $^{1}$, A. Damerau ${ }^{1,2}$, T. Gaber ${ }^{1,2}$, F. Buttgereit ${ }^{1,2} .{ }^{1}$ Charité Universitaetsmedizin Berlin, Rheumatology and Clinical Immunology, Berlin, Germany; ${ }^{2}$ German Rheumatism Research Center, Rheumatism Research, Berlin, Germany; ${ }^{3}$ Charité Universitaetsmedizin Berlin, Venerology and Allergology, Berlin, Germany

Background: Age-related impairment of classical neutrophil functions is well described (Fortin, McDonald et al. 2008). However, experimental evidence for age-related alterations of neutrophil metabolic adaptation towards nutrient deprivation -a feature of neutrophil battlefields- remains elusive. Moreover, age-related differences in neutrophil metabolic adaptation may contribute to age-related pathologies such as atherosclerosis, cancer, and autoimmune diseases including rheumatoid arthritis.

Objectives: Therefore, we hypothesized that metabolic adaptation of human neutrophils to glucose deprivation is impaired with increasing age.

Methods: We isolated human peripheral CD15+ neutrophils from four healthy young donors (mean age: $23.4 \pm 2.7$ ) and four healthy donors with a mean age of $58.7 \pm 2.4$. First, we analyzed the survival of neutrophils either stimulated with PMA or left untreated and subsequently incubated for $0 \mathrm{~h}$ and $6 \mathrm{~h}$ under varying glucose concentrations $(0,0.5,1,5$, and $10 \mathrm{mM})$. To address this, we used 7-AAD staining and flow cytometry. Using Seahorse ${ }^{\mathrm{TM}}$ technology, we determined basal respiration, ATP-bound respiration, and maximal and spare capacity.

Results: We show that neutrophils (purity $>95 \%$ ) survived for 6 hours in vitro, independent of treatment with PMA or concentrations of glucose in the culture medium. With negligible differences between the various concentrations of glucose used, the percentage of living cells after $6 \mathrm{~h}$ was $95 \% \pm 2.5$ without PMA and $75 \% \pm 4.7$ with PMA stimulation. No differences were uncovered in this respect between the two age groups. However, Seahorse ${ }^{T M}$ technology revealed significant differences in basal, maximal, and spare respiratory capacity. Briefly, OCR (pmol/min/cell count) with respect to basal, maximal and reserve respiratory capacity was lower in the elderly donors compared to the young donors. For instance, with a concentration of $5 \mathrm{mM}$ glucose, the basal respiration (OCR) was $17 \pm 0.7$ in elderly donors compared to $22.5 \pm 1$ in young donors, while the maximal respiration was $25 \pm 0.8$ in elderly and $41 \pm 0.6$ in the young donor group. Interestingly, these differences were independent of glucose concentration in the medium.

Conclusion: Our data show that basal metabolic parameters differ between neutrophils from young and older donors. Further experiments are needed to understand in detail the mechanisms and effects of age-related differences in metabolism on neutrophil functions.

REFERENCES:

[1] Fortin, C. F., P. P. McDonald, O. Lesur and T. Fülöp, Jr. (2008). "Aging and neutrophils: there is still much to do." Rejuvenation Res 11(5): 873-882.

Disclosure of Interests: None declared

DOI: 10.1136/annrheumdis-2021-eular.2018

\begin{tabular}{|l|l|}
\hline AB0032 & DIFFERENTIAL IMPACT OF BIOLOGICS AND \\
GLUCOCORTICOIDS ON TNF SECRETION AND \\
CD14+CD16+ MONOCYTES PERCENTAGE IN \\
CULTURE DERIVED FROM SYNOVIAL FLUIDS OF \\
PATIENTS WITH PSORIATIC ARTHRITIS-IN VITRO \\
RESEARCH
\end{tabular}

S. Gertel ${ }^{1}$, A. Polachek ${ }^{1}$, V. Furer ${ }^{1}$, D. Levartovsky ${ }^{1}$, R. Tzemach ${ }^{1}$, K. Meridor ${ }^{1}$, D. Caspi ${ }^{1}$, O. Elkayam ${ }^{1} .{ }^{1}$ Tel Aviv Sourasky Medical Center, Department of Rheumatology and the Sackler Faculty of Medicine, Tel Aviv University, Tel Aviv, Israel

Background: Inflammatory joint diseases, such as psoriatic arthritis (PsA), are frequently treated by biologics. Assessment of treatment efficacy is based upon change in clinical activity scores and in tender and swollen joint counts. Although the response to these agents may be attributed to their central anti-inflammatory effects, synovial response may be operating in parallel. The potential beneficial role of intra-articular injection of tumor necrosis factor (TNF) blockers compared to glucocorticoids (GCs) in reducing synovitis was shown by means of clinical and instrumental validated measures (1). The inflamed synovial fluid is rich in mononuclear cells, however, the different mode of action of the in vitro response of these cells to drugs may contribute to the understanding of cellular response to therapeutic agents in central as opposed to peripheral compartments.

Objectives: To evaluate the effect of biologics used in the management of PsA on synovial fluid mononuclear cells (SFMCs) in vitro, and to compare their modes of action to GCs that are used to locally alleviate synovial inflammation.

Methods: SFMCs were obtained from PsA patients $(n=11)$ during therapeutic knee arthrocentesis. The cells were cultured in vitro for 7 days in the presence of biologics (adalimumab, infliximab, secukinumab and ustekinumab, 10ug/ml) and
GCs (betamethasone and methylprednisolone, $1 \mathrm{ug} / \mathrm{ml}$ and 10ug/ml) or medium as control. Levels of the secreted TNF were measured by ELISA. Changes in $\% \mathrm{CD} 14^{+} \mathrm{CD} 16^{+}$monocytes were analyzed by flow cytometry.

Results: Both TNF inhibitors (adalimumab $p<0.01$, infliximab $p=0.0003$ ) and GCs (betamethasone and methylprednisolone at $1 \mathrm{ug} / \mathrm{ml} p<0.01$ and at $10 \mathrm{ug} / \mathrm{m}$ $p<0.04)$ significantly reduced TNF levels in cultured media derived from SFMCs of PsA patients $(n=8)$ compared to medium. None of the other biologics reduced TNF levels in culture (Fig. 1A). Additionally, \%CD14 ${ }^{+} \mathrm{CD} 16^{+}$SFMCs derived from PsA patients $(n=11)$ were significantly reduced by TNF inhibitors $(p=0.0003)$ compared to medium, however, other biologics and GCs did not display similar activity (Fig. 1B)
A

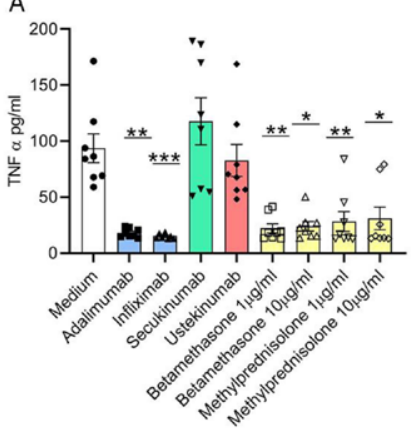

B

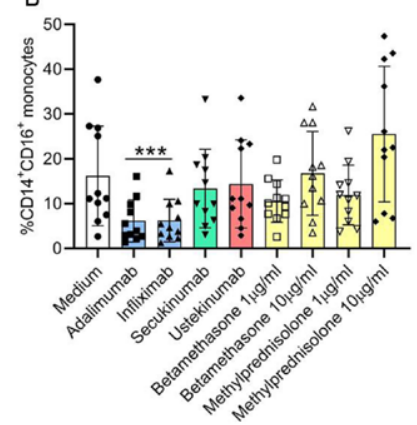

Figure 1. Both TNF inhibitors and GCs block TNF secretion but exhibit different activity on inflammatory $\mathrm{CD}_{14} 4^{+} \mathrm{CD} 16^{+}$monocytes derived from SFMCs of PsA patients. SFMCs were co-cultured for 7 days in the presence of adalimumab, infliximab, secukinumab and ustekinumab at $10 \mathrm{ug} / \mathrm{ml}$ or with betamethasone and methylprednisolone at $1 \mathrm{ug} / \mathrm{ml}$ and $10 \mathrm{ug} / \mathrm{ml}$ Medium alone was used as a control. (A) Culture supernatants were analyzed for TNF levels by ELISA $(n=8)$. (B) Cells were analyzed for $\% C D 14^{+} C D 16^{+}$monocytes by flow cytometry $(n=11)$. All $p$ values were calculated by the non-parametric one-way ANOVA Kruskal-Wallis test and Dunn's multiple comparison test, ${ }^{*} p<0.04,{ }^{\star *} p<0.01$ and ${ }^{* \star *} p=0.0003$.

Conclusion: Our data demonstrated marked activity mediated by TNF inhibitors in comparison with other biologics tested for their ability to suppress TNF secretion and inflammatory $\mathrm{CD} 14^{+} \mathrm{CD} 16^{+}$monocytes. In contrast, GCs suppressed TNF secretion but did not significantly change the proportion of inflammatory $\mathrm{CD} 14^{+} \mathrm{CD} 16^{+}$monocytes. These findings suggest an additional mechanism of action exerted directly by TNF inhibitors on synovial monocytes and which differs from that of GCs. These results warrant further studies of the therapeutic potential of local peripheral activity of TNF inhibitors for clinical application.

\section{REFERENCE:}

[1] Carubbi F, Zugaro L, Cipriani P, Conchiglia A, Gregori L, Danniballe C, et al. Safety and efficacy of intra-articular anti-tumor necrosis factor alpha agents compared to corticosteroids in a treat-to-target strategy in patients with inflammatory arthritis and monoarthritis flare. Int $\mathrm{J}$ Immunopathol Pharmacol. 2016;29:252-66.

Disclosure of Interests: None declared

DOI: 10.1136/annrheumdis-2021-eular.2064

\section{AB0033 MACROPHAGE ACTIVATION IN PATIENTS WITH ACTIVE RHEUMATOID ARTHRITIS AND SYSTEMIC LUPUS ERYTHEMATOSUS (PRELIMINARY DATA)}

E. Gerasimova ${ }^{1}$, T. Popkova ${ }^{1}$, T. Kirichenko ${ }^{2}$, A . Markin ${ }^{2}$, Y. Markina ${ }^{2}$, D. Gerasimova ${ }^{3}$, A. Bogatyreva ${ }^{2}$, A. Orekhov ${ }^{2} .{ }^{1}$ V.A. Nasonova Research Institute of Rheumatology, Laboratory of Systemic Rheumatic Diseases, Moscow, Russian Federation; ${ }^{2}$ Research Institute of Human Morphology, Laboratory of Cellular and Molecular Pathology of the Cardiovascular System, Moscow, Russian Federation; ${ }^{3}$ I.M. Sechenov First Moscow State Medical University (Sechenov University), Department of Pharmaceutical Economics, Moscow, Russian Federation

Background: The study of the ability of monocytes to activate associated with the clinical activity of immunological markers of inflammation in rheumatoid arthritis (RA) and systemic lupus erythematosus (SLE) will provide important and fundamentally new information on the involvement of these cells in the development of autoimmune rheumatic diseases (ARDs).

Objectives: To study macrophage activation in rheumatoid arthritis (RA) and systemic lupus erythematosus (SLE) patients (pts).

Methods: A total of 21 active ARDs pts (11 RA, 10 SLE) were enrolled in the study (median age was 55[44; 63] years; disease duration was 8 [2; 14] months). There are 11 pts with early RA (disease duration was $\leq 12$ months), moderate to high activity (DAS28 was 6.1[4.9;6.7]; SDAI was 25(22;31); ACCP was positive in $73 \%$ and RF in $87 \%$ cases) and 10 pts with active SLE (SLADAI-2K was $7[6 ; 8]$ ) 
in the study. All early RA pts and 4 SLE pts were not treated with glucocorticoids and disease-modifying antirheumatic drugs. Six SLE pts received low-dose glucocorticoids and hydroxychloroquine.

All pts were assessed for macrophage activation and laboratory data: ESR, RF, ACCP, CRP, ANA, anti-dsDNA. Isolation of monocytes was carried out according to the standard procedure for obtaining a leukocyte fraction in a Ficoll gradient and subsequent selection of CD14 + cells using magnetic separation. After isolation, the cells were cultured in X-Vivo medium. To assess the degree of monocyte activation, cells were stimulated by the addition of LPS. The value of monocyte activation was expressed as a ratio of the level of secretion of proinflammatory cytokines by monocytes cultured with and without LPS addition. Secretion levels were determined by ELISA. The belonging of the isolated cells to CD14 + monocytes was additionally confirmed by flow cytometry.

Results: Macrophage activation was $2.6(2.0 ; 5.4)$ and $4.8(2.8 ; 7.3)$ in RA and SLE pts, respectively $(p>0.05)$. In RA and SLE pts macrophage activation was independent of age, sex, body mass index, traditional risk factors (arterial hypertension, overweight, smoking, family history of cardiovascular diseases), RA activity scores (DAS28, SDAI), and SLADAI-2K. No association was found between macrophage activation and levels of ESR, RF, ACCP, CRP, ANA, and anti-dsDNA.

Conclusion: No differences in macrophage activation were found in RA and SLE pts. Macrophage activation was independent of age, sex, traditional risk factors, and ARD-related parameters. A study on a larger number of pts will clarify the link between macrophage activation and autoimmune disorders.

This work was supported by the Russian Science Foundation (Grant № 21-15-00225).

Disclosure of Interests: None declared

DOI: 10.1136/annrheumdis-2021-eular.2285

\section{AB0034 PD-1 AGONISM INHIBITS ACTIVATION OF PLASMACYTOID DENDRITIC CELLS}

I. Banerjee ${ }^{1}$, L. Edwards ${ }^{1}$, P. Halvey ${ }^{1}$, S. Alioto ${ }^{1}$, D. Cluckley ${ }^{1}$, C. Mitchell ${ }^{1}$ C. Cox ${ }^{1}$, E. Lurier ${ }^{1}$, M. Cianci ${ }^{1}$, S. Bengeri ${ }^{1}$, S. Borthakur ${ }^{1}$, K. Kis-Toth ${ }^{1}$, N. Higginson-Scott ${ }^{1}$, J. Viney ${ }^{1}$, K. L. Otipoby ${ }^{1} .{ }^{1}$ Pandion Therapeutics, Immunology, Watertown MA, United States of America

Background: T cell function is regulated by complex signaling networks of interconnected activators and inhibitors. Blockade of inhibitory receptors such as programmed death-1 (PD-1) has emerged as a novel treatment for multiple forms of cancer. One of the most common adverse events associated with blockade of the endogenous PD-1/PD-L1 pathway is the induction of autoimmune pathology in multiple tissues, demonstrating that PD-1 activation is necessary for normal immune homeostasis in humans (Kostine, et al., 2018). Given this body of clinical data, we sought to develop a PD-1 agonist antibody as a therapeutic approach to restore immune homeostasis in patients living with autoimmune diseases. PD-1 expression and function has been primarily described on T cells (Ishida, et al., 1992), with additional data available from several other immune cell populations (Ohaegbulam, et al., 2015).

Objectives: To study the effect of PD-1 agonism on plasmacytoid dendritic cell (pDC) function.

Methods: Human PBMCs stimulated with or without toll-like receptor (TLR)-9 agonist, CpG were analyzed by flow cytometry for PD-1 expression on immune cell subsets. To assess the impact of PD-1 agonist on pDC function human PBMCs were activated by $\mathrm{CpG}$ in the presence or absence of PD-1 agonist. Type-I interferon (IFN) levels were quantified using ELISA from culture supernatants. The expression of interferon stimulated genes was analyzed by qPCR as a measure of type-I IFN activation.

Results: We have discovered that TLR9 activation can induce PD-1 expression on plasmacytoid dendritic cells, which has not been previously reported. Further, we have demonstrated that PD-1 agonism inhibits TLR9-mediated activation and the effector functions of plasmacytoid dendritic cells.

Conclusion: These data suggest the potential of PD-1 as a target for regulating diseases with pathology generated by type-I IFN.

REFERENCES:

[1] Ishida, Y., Agata, Y., Shihibahara, K., \& Honjo, T. (1992). Induced expression of PD-1, a novel member of the immunoglobulin gene superfamily, upon programmed cell death. EMBO J., 11(11):3887-95.

[2] Kostine, M., Rouxel, L., Barnetche, T., Veillon, R., Martin, F., Dutriaux, C.,

Schaeverbeke, T. (2018). Rheumatic disorders associated with immune checkpoint inhibitors in patients with cancer-clinical aspects and relationship with tumour response: a single-centre prospective cohort study. Annual Rheumatic Disease, 77(3):393-398.

[3] Ohaegbulam, K. C., Assal, A., Lazar-Molnar, E., Yao, Y., \& Zang, X. (2015). Human cancer immunotherapy with antibodies to the PD-1 and PD-L1 pathway. Trends in Molecular Medicine, 21(1); 24-33.

Disclosure of Interests: Ishita Banerjee Shareholder of: Pandion Therapeutics, Employee of: Pandion Therapeutics, Lindsay Edwards Shareholder of: Pandion Therapeutics, Employee of: Pandion Therapeutics, Patrick Halvey Shareholder of: Pandion Therapeutics, Employee of: Pandion Therapeutics, Salvatore Alioto
Shareholder of: Pandion Therapeutics, Employee of: Pandion Therapeutics, David Cluckley Shareholder of: Pandion Therapeutics, Employee of: Pandion Therapeutics, Caitlin Mitchell Shareholder of: Pandion Therapeutics, Employee of: Pandion Therapeutics, Christopher Cox Shareholder of: Pandion Therapeutics, Employee of: Pandion Therapeutics, Emily Lurier Shareholder of: Pandion Therapeutics, Employee of: Pandion Therapeutics, Michael Cianci Shareholder of: Pandion Therapeutics, Employee of: Pandion Therapeutics, Soumya Bengeri Shareholder of Pandion Therapeutics, Employee of: Pandion Therapeutics, Susmita Borthaku Shareholder of: Pandion Therapeutics, Employee of: Pandion Therapeutics, Katalin Kis-Toth Shareholder of: Pandion Therapeutics, Employee of: Pandion Therapeutics, Nathan Higginson-Scott Shareholder of: Pandion Therapeutics, Consultant of: Biotech Companies, Employee of: Pandion Therapeutics, Jo Viney Shareholde of: Pandion Therapeutics, Employee of: Pandion Therapeutics, Kevin L. Otipoby Shareholder of: Pandion Therapeutics, Employee of: Pandion Therapeutics DOI: 10.1136/annrheumdis-2021-eular.2682

\section{Osteo arthritis, aetiology, pathology and animal models}

\section{$\mathrm{AB} 0035$}

A REVIEW OF THE CLINICAL AND ECONOMIC BURDEN OF OSTEOARTHRITIS PAIN BY SEVERITY IN THE UNITED STATES

A. Sadosky ${ }^{1}$, P. Schepman ${ }^{2}$, S. Thakkar ${ }^{2}$, R. Robinson ${ }^{3}$, C. Beck ${ }^{4} .{ }^{1}$ Apperture LLC, HEOR, Westerly, United States of America; ${ }^{2}$ Pfizer Inc, PHI, New York, United States of America; ${ }^{3}$ Eli Lilly and Company, Global Patient Outcomes \& Real World Evidence, Indianapolis, United States of America; ${ }^{4}$ Pfizer Inc, Medical Affairs, Tadworth, United Kingdom

Background: The development of new therapies to treat symptomatic osteoarthritis (OA) often requires targeting patient subgroups such as mild and/or moderate and/ or severe. Multiple assessments for pain are used in clinical and research settings, yet to quantify patient burden with increasing pain severity it is important to under stand the potential variability in outcomes based on definitions of severity used ${ }^{1}$. Objectives: The objective of this study was to examine studies in the published literature that report the burden of OA pain by severity to assess similarities and/ or differences across study methodologies and outcomes.

Methods: A targeted literature review of PubMed and Google Scholar was conducted January 2021 and included search terms: osteoarthritis, severity, United States (US), burden, quality of life, medication/treatment, and healthcare resource utilization. The search was limited to the English language, full-text articles, and no restriction on publication date. Results included a recent study of the burden of symptomatic OA pain respondents by severity level in the $\mathrm{US}^{2,3}$. Over 100 publication titles were reviewed. Comparison of findings was descriptive in nature.

Results: Nine publications were identified representing 7 unique studies, 6 being patient and/or healthcare provider surveys. Two studies focused on OA severity: the remaining 5 stratified patients by pain severity, and all but 2 of the 5 identified and confirmed pain as OA-related. Pain measures included numeric rating scales (generic 0-10, Western Ontario and McMaster Universities Arthritis Index [WOMAC] NRS 3.1), visual analog scales (generic 0-100, Short-Form McGill Pain Questionnaire Visual Analog Scale [SF-MPQ-VAS]) or Pain Interference with Activities (PIA) scale derived from the 12-Item Short Form Health Survey [SF-12v2] developed for the Medical Outcomes Study, with recall periods varying from 48 hours to 7 days to 4 weeks. Only one study exclusively assessed symptomatic patients only i.e., patients with pain scores of 0 were excluded; the remainder compared cohorts of no/mild pain with increasing severity cohorts. Four of the 7 studies examined pairwise differences among mild, moderate, and severe patients (1 study vs. a non-OA cohort); 2 compared no/mild vs. moderate-to-severe OA pain and 1 study compared mild to moderate-to-severe OA pain. For most outcomes examined like clinical comorbidities, quality of life, and healthcare resource utilization, increasing burden was observed with increasing $O A$ and/or pain severity despite study variability. Conclusion: Pain severity levels represent an important and distinguishing factor that contributes to health outcomes in OA patients in the US. Considerable heterogeneity across studies may impact how OA pain is defined, perceived by patients, and treated. Selecting appropriate OA pain severity assessments, including cut-points, may contribute to the successful monitoring of outcomes or comparisons of therapies to manage symptomatic OA pain, especially those that target specific pain severity subgroups.

\section{REFERENCES:}

[1] Hawker GA, Mian S, Kendzerska T et al. Arthritis Care and Research. 2011 63(11):S240-S252.

[2] Schepman P, Robinson RL, Thakkar S, et al. International Society of Pharmacoeconomics and Outcomes Research (ISPOR) Virtual Annual Meeting; May 2020.

[3] Schepman P, Thakkar S, Robinson RL, et al. PAINWeek 2020 Virtual Meeting; September 2020 